\title{
Ondelettes et poids de Muckenhoupt
}

\author{
par \\ PIERRE GILLES LEMARIÉ-RIEUSSET (Orsay)
}

\begin{abstract}
We study, for a basis of Hölderian compactly supported wavelets, the boundedness and convergence of the associated projectors $P_{j}$ on the space $L^{p}(d \mu)$ for some $p$ in $] 1, \infty[$ and some nonnegative Borel measure $\mu$ on $\mathbb{R}$. We show that the convergence properties are related to the $A_{p}$ criterion of Muckenhoupt.
\end{abstract}

Introduction. Les bases d'ondelettes sont des bases orthonormées de $L^{2}(\mathbb{R}, d x)$ mais elles sont également des bases inconditionnelles de nombreux espaces fonctionnels. Nous nous proposons d'étudier dans cet article les propriétés des bases d'ondelettes dans les espaces $L^{p}(d \mu)$.

Nous considérons une base d'ondelettes höldériennes à support compact telles qu'en a construites I. Daubechies. En particulier, le projecteur orthogonal $P_{j}$ de $L^{2}(\mathbb{R}, d x)$ sur l'espace $V_{j}$ de l'analyse multi-résolution sousjacente vérifie que si $f$ est continue à support compact alors $P_{j} f$ est encore continue à support compact; de plus, lorsque $j$ tend vers $\infty, P_{j} f$ converge uniformément vers $f$ et reste, pour $j \geq 0$, à support dans un compact fixe, de sorte que, pour toute mesure borélienne positive $\mu$ sur $\mathbb{R}$ et tout $p \in[1, \infty[$ on a, pour $f$ continue à support compact, $\lim _{j \rightarrow \infty} \int\left|P_{j} f-f\right|^{p} d \mu=0$.

Le but de cet article est de démontrer le théorème suivant :

ThÉORÈme 1. (a) Soit $\left(V_{j}\right)_{j \in \mathbb{Z}}$ une analyse multi-résolution d'I. Daubechies (à ondelettes höldériennes à support compact), $\mu$ une mesure borélienne positive sur $\mathbb{R}$ et $p \in] 1, \infty[$. Alors les assertions suivantes sont équivalentes :

(A1) les $P_{j}$ sont continus sur $L^{p}(d \mu)$ et on a, pour tout $f \in L^{p}(d \mu)$,

$$
\lim _{j \rightarrow \infty}\left\|f-P_{j} f\right\|_{L^{p}(d \mu)}=0 \quad \text { et } \quad \lim _{j \rightarrow-\infty}\left\|P_{j} f\right\|_{L^{p}(d \mu)}=0 ;
$$

(A2) $\quad d \mu=v(x) d x$ où $v$ appartient à la classe $A_{p}$ de Muckenhoupt;

(A3) les ondelettes $\left(\psi_{j, k}\right)(j \in \mathbb{Z}, k \in \mathbb{Z})$ forment une base inconditionnelle de $L^{p}(d \mu)$. 
(b) Pour $p=1$, les assertions suivantes sont équivalentes :

(B1) les opérateurs $P_{j}, j \in \mathbb{Z}$, sont équicontinus sur $L^{1}(d \mu)$;

(B2) $d \mu=v(x) d x$ où $v$ appartient à la classe $A_{1}$ de Muckenhoupt.

Rappelons la définition des classes $A_{p}$ de Muckenhoupt. Un poids $v(x)$ sur $\mathbb{R}$ (c'est-à-dire une fonction localement intégrable pour la mesure de Lebesgue et positive) appartient à la classe $A_{p}$ de Muckenhoupt $(1 \leq p<\infty)$ s'il existe une constante $C \geq 1$ telle que pour tout intervalle $I$ (de longueur $|I|)$ on ait

$$
\begin{gathered}
\frac{1}{|I|}\left(\int_{I} v d x\right)^{1 / p}\left(\int_{I} v^{-1 /(p-1)} d x\right)^{(p-1) / p} \leq C \quad \text { si } p>1, \\
\frac{1}{|I|} \int_{I} v d x \leq C \underset{x \in I}{\operatorname{essinf}} v(x) \quad \text { si } p=1 .
\end{gathered}
$$

Notation. Pour $\mu$ mesure borélienne positive sur $\mathbb{R}, \quad p \in[1, \infty[$ et $f$ borélienne bornée à support compact on notera

$$
\begin{aligned}
\||f|\|_{p, \mu} & =\left(\int|f|^{p} d \mu\right)^{1 / p}, \\
\||f|\|_{p, \mu, *} & =\sup \left\{\left|\int f g d x\right|: g \text { continue à support compact, }\|g\|_{p, \mu} \leq 1\right\} .
\end{aligned}
$$

On remarquera que $\|f \mid\|_{p, \mu, *}<\infty$ si et seulement si il existe $h \in$ $L^{p /(p-1)}(d \mu)$ telle que les mesures $f d x$ et $h d \mu$ soient égales. Lorsque $d \mu=$ $v d x$, on notera ||$|f| \|_{p, v}$ et $\||f|\|_{p, v, *}$ au lieu de $\||| f \mid\|_{p, v d x}$ et $\||f|\|_{p, v d x, *}$. On a alors, si $p>1$,

$$
\||f|\|_{p, v, *}=\left(\int|f(x)|^{p /(p-1)} v(x)^{-1 /(p-1)} d x\right)^{(p-1) / p}
$$

et

Par conséquent,

$$
\|\mid f\|_{1, v, *}=\operatorname{ess} \sup \frac{|f(x)|}{|v(x)|} .
$$

$$
v \in A_{p} \quad \operatorname{ssi} \quad \sup _{I} \frac{1}{|I|}\left|\left\|\chi _ { I } \left|\left\|_ { p , v } \left|\left\|\chi_{I} \mid\right\|_{p, v, *}<\infty\right.\right.\right.\right.\right.
$$

(où $\chi_{I}$ désigne la fonction caractéristique de l'intervalle $I$ ).

Le théorème 1 repose essentiellement sur le théorème suivant :

ThÉORÈme 2. Soit $\left(V_{j}\right)_{j \in \mathbb{Z}}$ une analyse multi-résolution d'I. Daubechies (à ondelettes höldériennes à support compact), $\mu$ une mesure borélienne positive sur $\mathbb{R}$ et $p \in[1, \infty[$. Alors les assertions suivantes sont équivalentes :

(C1) $\quad C_{1}=\sup \left\{\left\|\left|P_{0} f\right|\right\|_{p, \mu}: f\right.$ continue à support compact, $\left.\|f \mid\|_{p, \mu} \leq 1\right\}$ $<\infty$

(C2) $\quad C_{2}=\sup _{|I|=1}\left|\left\|\chi_{I}\left|\left\|_{p, \mu}\left|\left\|\chi_{I} \mid\right\|_{p, \mu, *}<\infty\right.\right.\right.\right.\right.$. 
De plus, $C_{2}$ se majore en fonction seulement de $C_{1}$, de $p$ et du choix de

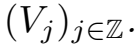

Le théorème 2 peut se paraphraser de la manière suivante :

Proposition 1. Soit $\left(V_{j}\right)$ une analyse multi-résolution d'I. Daubechies (à ondelettes höldériennes à support compact), $\mu$ une mesure borélienne positive sur $\mathbb{R}$ et $p \in[1, \infty[$. Alors :

(i) $P_{j}$ est continu sur $L^{p}(d \mu)$ pour (au moins un) $j \in \mathbb{Z}$ si et seulement si on a pour (au moins un) $R>0$,

$$
\left.\left.\sup _{|I|=R} \frac{1}{|I|}||\left|\chi_{I}\right|\right|_{p, \mu}||\left|\chi_{I}\right|\right|_{p, \mu, *}<\infty ;
$$

(ii) les $P_{j}$ sont continus sur $L^{p}(d \mu)$ et pour tout $f \in L^{p}(d \mu)$,

$$
\lim _{j \rightarrow \infty}\left\|\mid P_{j} f-f\right\|_{p, \mu}=0
$$

si et seulement si on a pour (au moins un) $R>0$,

$$
\left.\sup _{|I| \leq R} \frac{1}{|I|}||\left|\chi_{I}\right|||_{p, \mu}||\left|\chi_{I}\right|\right|_{p, \mu, *}<\infty .
$$

De plus, dans ce cas, la mesure $\mu$ est absolument continue par rapport à la mesure de Lebesgue : $d \mu=v d x$ avec $v$ localement intégrable.

(iii) Les $P_{j}, j \in \mathbb{Z}$, sont équicontinus sur $L^{p}(d \mu)$ si et seulement si $v \in$ $A_{p}$, ou encore,

$$
\sup _{I} \frac{1}{|I|}||\left|\chi_{I}\right|\left\|_{p, \mu}||\left|\chi_{I}\right|\right\|_{p, \mu, *}<\infty .
$$

La proposition 1 introduit donc des critères de Muckenhoupt à une échelle (cas (i)) ou local ou aux petites échelles (cas (ii)) en plus du critère global (1.3). Le critère local peut se révéler très utile dans la mesure où dans de nombreux problèmes on ne décompose pas les fonctions à toutes les échelles (sur tous les $W_{j}$ ) mais seulement sur les petites échelles ( $\operatorname{sur} V_{j_{0}}$ et sur les $\left.W_{j}, j \geq j_{0}\right)$.

1. Rappels sur les ondelettes de Daubechies. Pour tout $N \geq 2$, I. Daubechies a construit dans [1] une fonction $\varphi$ qui vérifie : $\varphi$ est de classe $C^{\alpha N}$ (où $\alpha>0$ ne dépend pas de $N$ ), à valeurs réelles et à support compact dans $\mathbb{R}$;

(2.2) les $\varphi(x-k), k \in \mathbb{Z}$, forment une famille orthonormée de $L^{2}(\mathbb{R}, d x)$;

(2.3) $\varphi(x / 2)=\sum_{k=0}^{2 N-1} a_{k} \varphi(x-k)$ avec $a_{0} \neq 0$ et $a_{2 N-1} \neq 0$.

La fonction $\varphi$ est alors la fonction d'échelle d'une analyse multi-résolution $\left(V_{j}\right)_{j \in \mathbb{Z}}$ au sens de S. Mallat [5]. Plus précisément, on définit :

$$
\varphi_{j, k}(x)=2^{j / 2} \varphi\left(2^{j} x-k\right)(j \in \mathbb{Z}, k \in \mathbb{Z}) ;
$$


(3.2) $\quad V_{j}$ est le sous-espace fermé de $L^{2}(\mathbb{R}, d x)$ engendré par les $\varphi_{j, k}, k \in \mathbb{Z}$; (3.3) $\quad P_{j} f(x)=\sum_{k \in \mathbb{Z}}\left\langle f \mid \varphi_{j, k}\right\rangle \varphi_{j, k}(x)$.

$P_{j}$ est le projecteur orthogonal de $L^{2}(\mathbb{R}, d x)$ sur $V_{j}(\langle f \mid g\rangle$ désigne le produit scalaire $\int_{-\infty}^{\infty} f(x) \bar{g}(x) d x$ dans $\left.L^{2}(\mathbb{R}, d x)\right)$. Dire que $\left(V_{j}\right)_{j \in \mathbb{Z}}$ est une analyse multi-résolution de fonction d'échelle $\varphi$, c'est par définition dire que $\left(V_{j}\right)$ vérifie :

(4.1) $\quad V_{j} \subset V_{j+1}, \bigcap_{j \in \mathbb{Z}} V_{j}=\{0\}, \bigcup_{j \in \mathbb{Z}} V_{j}$ est dense dans $L^{2}(\mathbb{R}, d x)$,

(4.2) $f(x) \in V_{j}$ ssi $f(2 x) \in V_{j+1}$,

(4.3) les $\varphi(x-k), k \in \mathbb{Z}$, forment une base orthonormée de $V_{0}$.

A la fonction d'échelle $\varphi$ est associée une ondelette $\psi$ définie par

$$
\psi(x)=\sum_{k=0}^{2 N-1}(-1)^{k} a_{2 N-1-k} \varphi(x-k) .
$$

On définit alors également :

(6.1) $\quad \psi_{j, k}(x)=2^{j / 2} \psi\left(2^{j} x-k\right)$;

(6.2) $W_{j}$ est le complémentaire orthogonal de $V_{j}$ dans $V_{j+1}$;

(6.3) $\quad Q_{j} f(x)=\sum_{k \in \mathbb{Z}}\left\langle f \mid \psi_{j, k}\right\rangle \psi_{j, k}$.

Alors les $\psi_{j, k}, k \in \mathbb{Z}$, forment une base orthonormée de $W_{j}$ et $Q_{j}=P_{j+1}-P_{j}$ est le projecteur orthogonal de $L^{2}(\mathbb{R}, d x)$ sur $W_{j}$. En particulier, les $\psi_{j, k}$ $(j \in \mathbb{Z}, k \in \mathbb{Z})$ forment une base d'ondelettes de $L^{2}(\mathbb{R}, d x)$, c'est-à-dire une base orthonormée de $L^{2}(\mathbb{R}, d x)$ engendrée à partir d'une seule fonction $\psi$ par les translations-dilatations dyadiques (6.1).

Les principales propriétés de ces bases d'ondelettes sont décrites dans le livre de Y. Meyer [6]. Celles que nous utiliserons plus particulièrement sont les suivantes :

(7.1) $\quad \sum_{k \in \mathbb{Z}} \varphi(x-k)=1$;

(7.2) $\quad \int \psi d x=0$

(7.3) $\operatorname{Supp} \varphi=\operatorname{Supp} \psi=[0,2 N-1]$;

(7.4) si $\sum \lambda_{k} \varphi(x-k)$ est nulle sur un intervalle $I$ et si $\left|I \cap \operatorname{Supp} \varphi\left(x-k_{0}\right)\right|$ $>0$, alors $\lambda_{k_{0}}=0$.

Les propriétés (7.3) et (7.4) ont été démontrées récemment par G. Malgouyres [3], [4].

Remarque. De (7.1) on déduit effectivement la propriété, évoquée dans l'introduction, que $P_{j} f$ tend vers $f$ uniformément lorsque $f$ est continue à support compact :

$$
\left|P_{j} f(x)-f(x)\right| \leq \sum_{k \in \mathbb{Z}}\left|\varphi\left(2^{j} x-k\right)\right| \cdot\left|\left\langle f(y) \mid 2^{j} \varphi\left(2^{j} y-k\right)\right\rangle-f(x)\right|
$$




$$
\begin{aligned}
& \leq \sum_{k \in \mathbb{Z}}\left|\varphi\left(2^{j} x-k\right)\right| \cdot\left|\int(f(y)-f(x)) 2^{j} \varphi\left(2^{j} y-k\right) d y\right| \\
& \leq(2 N-1)^{2}\|\varphi\|_{\infty}^{2} \sup _{|x-y| \leq(2 N-1) / 2^{j}}|f(x)-f(y)| .
\end{aligned}
$$

2. Démonstration du théorème 2. Avant de démontrer le théorème 2 , remarquons la propriété suivante :

LEMME 1. On note

$$
\alpha_{0}=\sup _{|I|=1}\left|\left\|\chi_{I}||_{p, \mu}|| \chi_{I} \mid\right\|_{p, \mu, *}\right.
$$

et

$$
\alpha_{1}=\left.\sup _{k \in \mathbb{Z}}\||\varphi(x-k)|\|\right|_{p, \mu}\left\||\varphi(x-k) \||_{p, \mu, *}\right.
$$

(i) Si $\alpha_{0}<\infty$, il existe $\beta_{0}=4 \alpha_{0}^{2}$ tel que, pour tout $x \in \mathbb{R}$,

$$
\left.\frac{1}{\beta_{0}}||\left|\chi_{[x, x+1]}\right|\right|_{p, \mu} \leq\left|\left\|\chi_{[x+1, x+2]}||_{p, \mu} \leq \beta_{0}\right\|\right| \chi_{[x, x+1]} \mid \|_{p, \mu} .
$$

(ii) Si $\alpha_{1}<\infty$, il existe $\beta_{1}=\beta_{2}(\varphi) \alpha_{1}$ (où $\beta_{2}(\varphi)$ ne dépend que de $\varphi$ ) tel que, pour tout $k \in \mathbb{Z}$,

$$
\left.\frac{1}{\beta_{1}}\|\varphi(x-k)\|\right|_{p, \mu} \leq\|\mid \varphi(x-k-1)\|\left\|_{p, \mu} \leq \beta_{1}\right\| \varphi(x-k)\|\|_{p, \mu} .
$$

(iii) $\alpha_{0}<\infty$ est équivalent à $\alpha_{1}<\infty$ et $\alpha_{0}$ ne se majore qu'en fonction de $\alpha_{1}, p$ et $\varphi$ (et de même $\alpha_{1}$ en fonction de $\alpha_{0}, p$ et $\varphi$ ) :

$$
\alpha_{0} \leq 4 N^{2} \beta_{1}^{4 N-2} \alpha_{1} \quad \text { et } \quad \alpha_{1} \leq(2 N-1)^{2}\|\varphi\|_{\infty}^{2} \beta_{0}^{4 N-4} \alpha_{0} .
$$

Démonstration. (i) En effet, on a

$$
\int \chi_{[x, x+1]}(t) \chi_{[x+1 / 2, x+3 / 2]}(t) d t=\frac{1}{2}
$$

et donc

$$
\begin{aligned}
\left\|\chi_{[x, x+1]} \mid\right\|_{p, \mu}= & 4\left|\left\|\chi_{[x, x+1]} \mid\right\|_{p, \mu} \int \chi_{[x, x+1]} \chi_{[x+1 / 2, x+3 / 2]} d t\right. \\
& \times \int \chi_{[x+1 / 2, x+3 / 2]} \chi_{[x+1, x+2]} d t \\
\leq & 4\left|\left\|\chi _ { [ x , x + 1 ] } \left|\left\|_ { p , \mu } \left|\left\|\chi _ { [ x , x + 1 ] } \left|\left\|_ { p , \mu , * } \left|\left\|\chi_{[x+1 / 2, x+3 / 2]} \mid\right\|_{p, \mu}\right.\right.\right.\right.\right.\right.\right.\right.\right. \\
& \times\left\|| | \chi _ { [ x + 1 / 2 , x + 3 / 2 ] } \left|\left\|_ { p , \mu , * } \left|\left\|\chi_{[x+1, x+2]} \mid\right\|_{p, \mu}\right.\right.\right.\right. \\
\leq & 4 \alpha_{0}^{2}\left\|\chi_{[x+1, x+2]} \mid\right\|_{p, \mu}
\end{aligned}
$$

et de même ||$\left|\chi_{[x, x+1]}\right|\left\|_{p, \mu} \leq 4 \alpha_{0}^{2}\right\|\left|\chi_{[x-1, x]}\right| \|_{p, \mu}$. 
(ii) D'après $(7.3), \operatorname{Supp} \varphi(x) \varphi(x-1)=[1,2 N-1]$ et donc il existe $h$ continue à support compact telle que

$$
\int h(x) \varphi(x) \varphi(x-1) d x=1 \text {. }
$$

On a alors

$$
\begin{aligned}
\|\varphi(x-k) \mid\|_{p, \mu} & =\left|\|\varphi(x-k) \mid\|_{p, \mu} \int h(x-k) \varphi(x-k) \varphi(x-k-1) d x\right. \\
& \leq\left|\left\|\varphi ( x - k ) \left|\left\|_ { p , \mu } \left|\left\|\varphi ( x - k ) \left|\left\|_{p, \mu, *} \mid\right\| h(x-k) \varphi(x-k-1)\|\|_{p, \mu}\right.\right.\right.\right.\right.\right.\right. \\
& \leq \alpha_{1}\|h\|_{\infty}\|\varphi(x-k-1)\| \|_{p, \mu}
\end{aligned}
$$

et de même $\||\varphi(x-k)|\|_{p, \mu} \leq \alpha_{1}\|h\|_{\infty}\||\varphi(x-k+1)|\|_{p, \mu}$.

(iii) En effet, $\operatorname{Supp} \varphi(x-k)=[k, k+2 N-1]$ et donc

$$
\begin{aligned}
\|\varphi(x-k)\| \|_{p, \mu} & \leq\|\varphi\|_{\infty} \sum_{p=0}^{2 N-2}\|\| \chi_{[k+p, k+p+1]}\|\|_{p, \mu} \\
& \leq(2 N-1) \beta_{0}^{2 N-2}\|\varphi\|_{\infty}\left\|\chi_{[k, k+1]}\right\| \|_{p, \mu}
\end{aligned}
$$

et

$$
\begin{aligned}
\|\varphi(x-k)\|_{p, \mu, *} & \leq\|\varphi\|_{\infty} \sum_{p=0}^{2 N-2}\left\|\chi_{[k+p, k+p+1]}\right\|_{p, \mu, *} \\
& \leq \alpha_{0}\|\varphi\|_{\infty} \sum_{p=0}^{2 N-2} \frac{1}{\left\|\chi_{[k+p, k+p+1]}\right\|_{p, \mu}} \\
& \leq \alpha_{0}(2 N-1) \beta_{0}^{2 N-2}\|\varphi\|_{\infty} \frac{1}{\left\|\chi_{[k, k+1]}\right\|_{p, \mu}}
\end{aligned}
$$

d'où $\alpha_{1} \leq\|\varphi\|_{\infty}^{2} \alpha_{0}(2 N-1)^{2} \beta_{0}^{4 N-4}$.

Inversement, si $k \leq x<k+1$, alors

$$
\chi_{[x, x+1]}(t)=\chi_{[x, x+1]}(t) \sum_{p=-1}^{2 N-2} \varphi(t-k+p)
$$

et donc

$$
\left\|\chi_{[x, x+1]}\left|\left\|_{p, \mu} \leq \sum_{p=-1}^{2 N-2}\right\|\right| \varphi(t-k+p)\right\|\left\|_{p, \mu} \leq 2 N \beta_{1}^{2 N-1}\right\| \mid \varphi(t-k-1)\|\|_{p, \mu}
$$

et

d'où $\alpha_{0} \leq \alpha_{1} 4 N^{2} \beta_{1}^{4 N-2}$.

$$
\begin{aligned}
\left\|\chi_{[x, x+1]} \mid\right\|_{p, \mu, *} & \leq \alpha_{1} \sum_{p=-1}^{2 N-2} \frac{1}{\|\varphi(t-k+p)\| \|_{p, \mu, *}} \\
& \leq 2 N \alpha_{1} \beta_{1}^{2 N-1} \frac{1}{\|\varphi(t-k-1)\|_{p, \mu}},
\end{aligned}
$$


Démontrer le théorème 2 revient alors à démontrer le théorème suivant :

ThÉorÈme 2bis. Pour $p \in\left[1, \infty\left[\right.\right.$ et $C \geq 1$ on note $K_{C, p}$ l'ensemble des mesures boréliennes positives $\mu$ sur $\mathbb{R}$ telles que:

(i) $\int|\varphi(x)|^{p} d \mu(x)=1$;

(ii) pour toute fonction $f$ continue à support compact,

$$
\int\left|P_{0} f\right|^{p} d \mu \leq C \int|f|^{p} d \mu
$$

Alors $K_{C, p}$, muni de la topologie de la convergence vague, est un compact métrisable et

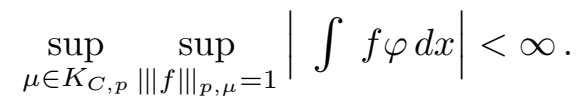

Pour démontrer ce théorème, nous allons recourir à une série de lemmes intermédiaires. Dans ce qui suit, le lettre $C^{\prime}$ désignera diverses constantes dépendant de $C, p$ et $\varphi$ mais pas de $\mu$.

LEMME 2. || $\mid \varphi(x-k) \|_{p, \mu} \leq C^{\prime|k|}$ pour tout $k \in \mathbb{Z}$ et toute $\mu \in K_{C, p}$.

En effet, on peut trouver une fonction $h_{0}$ continue à support compact telle que

$$
\int h_{0}(x) \varphi(x) \varphi(x-p) d x=\delta_{1, p}
$$

(où $\delta_{1, p}=1$ si $p=1$, et 0 si $p \neq 1$ ). Cela provient du fait que si

$$
\sum_{p=-2 N+2}^{2 N-2} \alpha_{p} \varphi(x) \varphi(x-p)=0 \quad \text { p.p. }
$$

alors $\sum \alpha_{p} \varphi(x-p)$ est nulle p.p. sur un ouvert dense de [0,2N-1] (puisque $\operatorname{Supp} \varphi=[0,2 N-1]$ et donc que $\varphi(x) \neq 0$ sur un ouvert dense de $] 0,2 N-1])$ et donc $\sum \alpha_{p} \varphi(x-p)$ est nulle sur [0,2N-1]; par (7.4) on obtient que tous les $\alpha_{p}$ sont nuls. Les formes linéaires $h \rightarrow \int h \varphi(x) \varphi(x-p) d x$ (pour $-2 N+2 \leq p \leq 2 N-2$ ) sont donc linéairement indépendantes sur l'espace des fonctions continues (nulles à l'infini) et un lemme classique assure l'existence de $h_{0}$ (qu'on peut supposer à support compact puisque seules ses valeurs sur $[0,2 N-1]$ interviennent). Mais alors on a

$$
\varphi(x-k-1)=P_{0}\left(h_{0}(x-k) \varphi(x-k)\right)
$$

et

d'où

$$
\varphi(x+k+1)=P_{0}\left(h_{0}(x+k+1) \varphi(x+k)\right),
$$

$$
\left|\left\|\varphi(x-k-1)\left|\left\|_{p, \mu} \leq C\right\| h_{0}\left\|_{\infty}\right\| \varphi(x-k)\right|\right\|_{p, \mu}\right.
$$

et de même

$$
\|\| \varphi(x+k+1) \mid\left\|_{p, \mu} \leq C\right\| h_{0}\left\|_{\infty}\right\|\|\varphi(x+k)\| \|_{p, \mu} .
$$


Lemme 3. $\forall \mu \in K_{C, p}, \forall n \in \mathbb{N}, \mu([-n, n]) \leq C^{\prime n}$.

Cela provient directement de (7.1) et du lemme 2 :

$$
\begin{aligned}
\mu([-n, n]) & =\int_{[-n, n]}\left|\sum_{k} \varphi(x-k)\right|^{p} d \mu \\
& \leq\left(\left.\sum_{k=-n-2 N+2}^{n-1}\|\varphi(x-k)\|\right|_{p, \mu}\right)^{p} \\
& \leq\left(\sum_{k=-n-2 N+2}^{n-1} C^{\prime|k|}\right)^{p} \leq C^{\prime n p}\left(2 \sum_{k=0}^{\infty} \frac{1}{C^{\prime k}}\right)^{p} \leq C^{\prime \prime n} .
\end{aligned}
$$

Lemme $4 . K_{C, p}$ est un compact métrisable pour la topologie de la convergence vague.

La topologie de la convergence vague est définie sur l'espace des mesures boréliennes par les semi-normes $\|\mu\|_{f}=\left|\int f d \mu\right|$ où $f$ décrit l'espace des fonctions continues à support compact. On note $B_{n}$ la boule fermée de centre 0 et de rayon $C^{\prime n}$ (où $C^{\prime}$ est la constante du lemme 3 ) dans l'espace des mesures boréliennes sur $[-n, n] ; B_{n}$ muni de la topologie de la convergence vague est un compact métrisable d'après le théorème de Banach-Alaoglu; or $K$ s'identifie à un fermé de $\prod_{n \in \mathbb{N}} B_{n}$ par l'application $\mu \rightarrow\left(\left.\mu\right|_{[-n, n]}\right)_{n \in \mathbb{N}}$. Le lemme est donc démontré.

Lemme 5. $\forall \mu \in K_{C, p}, \operatorname{Supp} \mu=\mathbb{R}$.

En effet, considérons un intervalle $I$ borné tel que $|I|>0$. Fixons $k_{0} \in \mathbb{Z}$ tel que $\left|\left[k_{0}, k_{0}+2 N-1\right] \cap I\right|>0$. Le même argument que dans la démonstration du lemme 2 montre qu'il existe $h_{I}$ continue à support compact telle que $\int h_{I}(x) \chi_{I}(x) \varphi(x-k) d x=\delta_{k, k_{0}}$ (la mesure $\chi_{I}(x) \varphi\left(x-k_{0}\right) d x$ étant linéairement indépendante, d'après (7.4), des $\chi_{I} \varphi(x-k) d x$ avec $k \neq$ $\left.k_{0}\right)$. On a alors

$$
\left\|\varphi\left(x-k_{0}\right)\right\|\left\|_{p, \mu} \leq C\right\| h_{I} \|_{\infty} \mu(I)^{1 / p} .
$$

Or la démonstration du lemme 2 montre que

$$
|| \varphi\left(x-k_{0}\right)||_{p, \mu} \geq C^{\prime-\left|k_{0}\right|}>0
$$

et donc $\mu(I)>0$.

Lemme 6. On note $K=\left\{\left(\varepsilon_{k}\right)_{-2 N+2 \leq k \leq 2 N-2}: \sum\left|\varepsilon_{k}\right|^{p}=1\right\}$. Alors

$$
\inf _{\varepsilon \in K} \inf _{\mu \in K_{C, p}} \int_{[0,2 N-1]}\left|\sum_{k=-2 N+2}^{2 N-2} \varepsilon_{k} \varphi(x-k)\right|^{p} d \mu(x)>0 .
$$


Remarquons d'abord que l'application $F$ qui à $(\varepsilon, \mu) \in K \times K_{C, p}$ associe

$$
F(\varepsilon, \mu)=\int_{[0,2 N-1]}\left|\sum_{k} \varepsilon_{k} \varphi(x-k)\right|^{p} d \mu
$$

est continue : en effet, si $\left(\varepsilon_{n}, \mu_{n}\right)$ converge vers $(\varepsilon, \mu)\left(\mu_{n}\right.$ convergeant vers $\mu$ au sens de la convergence vague) alors

$$
\sum_{k=-2 N+2}^{2 N-2} \varepsilon_{k, n} \varphi(x-k)
$$

converge uniformément vers

$$
\sum_{k=-2 N+2}^{2 N-2} \varepsilon_{k} \varphi(x-k)
$$

comme, d'après le lemme $3, \sup _{n} \mu_{n}([0,2 N-1])<\infty$ il est clair que

$$
\lim _{n \rightarrow \infty} F\left(\varepsilon_{n}, \mu_{n}\right)-F\left(\varepsilon, \mu_{n}\right)=0 ;
$$

par ailleurs $F\left(\varepsilon, \mu_{n}\right)$ converge vers $F(\varepsilon, \mu)$. $F$ est donc continue; comme $K$ et $K_{C, p}$ sont compacts, la borne inférieure de $F$ sur $K \times K_{C, p}$ est atteinte. Il reste à montrer qu'elle est non nulle.

Supposons que $F(\varepsilon, \mu)$ soit nul; cela implique que

$$
\chi_{[0,2 N-1]}(x)\left(\sum_{k=-2 N+2}^{2 N-2} \varepsilon_{k} \varphi(x-k)\right)
$$

soit nulle $\mu$-presque partout; or la fonction $\sum \varepsilon_{k} \varphi(x-k)$ est continue; si elle était non nulle en un point de ]0, $2 N-1$ [, elle serait non nulle sur un (petit) intervalle $I$ autour de ce point; or d'après le lemme $5, \mu(I)>0$; on obtient donc que $\sum \varepsilon_{k} \varphi(x-k)$ doit être nulle sur [0,2N-1] et (7.4) entraîne alors que les $\varepsilon_{k}$ sont nuls pour $-2 N+2 \leq k \leq 2 N-2$; ce dernier résultat est absurde puisque $\sum \varepsilon_{k}^{p}=1$. $F(\varepsilon, \mu)$ est donc non nul. Le lemme 6 est alors démontré.

Le théorème 2 bis est alors immédiat. Appelons $\alpha$ la borne inférieure de $F(\varepsilon, \mu)$ sur $K \times K_{C, p}$. Alors on a

$$
\begin{aligned}
|\langle f \mid \varphi\rangle| & \leq\left(\sum_{k=-2 N+2}^{2 N-2}|\langle f \mid \varphi(x-k)\rangle|^{p}\right)^{1 / p} \\
& \leq\left(\frac{1}{\alpha} \int_{[0,2 N-1]}\left|\sum\langle f \mid \varphi(x-k)\rangle \varphi(x-k)\right|^{p} d \mu\right)^{1 / p} \\
& \leq \alpha^{-1 / p}\left\|\left|P_{0} f\right|\right\|_{p, \mu} \leq C \alpha^{-1 / p}\left|\|f \mid\|_{p, \mu} .\right.
\end{aligned}
$$

Le théorème 2 bis est donc démontré. 
Nous pouvons maintenant démontrer le théorème 2. Supposons que

$$
C_{1}=\sup _{\mid\|f\|_{p, \mu}=1}\left\|P_{0} f\right\|_{p, \mu}<\infty .
$$

Remarquons qu'alors tous les $\left|\|\varphi(x-k) \mid\|_{p, \mu}, k \in \mathbb{Z}\right.$, sont non nuls (si $\mu$ est non nulle) : il suffit de reprendre les démonstrations des lemmes 2 et 3 pour voir que si $\varphi=0$ dans $L^{p}(d \mu)$ et si $P_{0}$ est continu sur $L^{p}(d \mu)$ alors tous les $\varphi(x-k), k \in \mathbb{Z}$, sont nuls et pour finir $\mu$ est nulle. On remarque alors que $\mu_{k}$ définie par

$$
\int f(x) d \mu_{k}=\int f(x-k) \frac{d \mu}{\|\varphi(x-k)\|_{p, \mu}^{p}}
$$

vérifie que $\mu_{k} \in K_{C_{1}, p}$ et donc $\|\varphi\|_{p, \mu_{k}, *} \leq C^{\prime}$ d'après le théorème 2bis; or $\|\varphi \varphi\|_{p, \mu_{k}, *}=\left.\left\|\left.|\varphi(x-k)|\right|_{p, \mu}\right\||\varphi(x-k)|\right|_{p, \mu, *}$.

On utilise alors le lemme 1 pour conclure que

$$
C_{2}=\sup _{|I|=1}\left\|\left|\chi_{I}\right|\right\|_{p, \mu}|| \chi_{I} \mid \|_{p, \mu, *}
$$

est fini et se majore en fonction de $C_{1}, p$ et $\varphi$, mais indépendamment de $\mu$.

Réciproquement, si $C_{2}<\infty$, alors $C_{3}=\sup \|\varphi(x-k)\|_{p, \mu} \times$ $\||\varphi(x-k)|\|_{p, \mu, *}$ est fini (toujours d'après le lemme 1 ). On a alors

$$
\begin{aligned}
\left\|P_{0} f\right\|_{p, \mu} \leq & \sum_{r=1}^{2 N-1} \| \sum_{k \in \mathbb{Z}}\langle f \mid \varphi(x-k(2 N-1)-r)\rangle \\
& \times \varphi(x-k(2 N-1)-r) \mid \|_{p, \mu} \\
\leq & \sum_{r=1}^{2 N-1}\left(\sum_{k \in \mathbb{Z}}|\langle f \mid \varphi(x-k(2 N-1)-r)\rangle|^{p}\right. \\
& \left.\times\|\varphi(x-k(2 N-1)-r)\|_{p, \mu}^{p}\right)^{1 / p}
\end{aligned}
$$

$(\operatorname{car} \varphi(x)$ et $\varphi(x-k(2 N-1))$ sont à supports disjoints $)$ et donc

$$
\begin{aligned}
\left\|P_{0} f\right\|_{p, \mu} \leq & (2 N-1)^{(p-1) / p}\left(\sum_{k \in \mathbb{Z}}|\langle f \mid \varphi(x-k)\rangle|^{p}\||\varphi(x-k)|\|_{p, \mu}^{p}\right)^{1 / p} \\
\leq & (2 N-1)^{(p-1) / p} \\
& \times\left(\sum_{k \in \mathbb{Z}}\left\|\left|f \chi_{[k, k+2 N-1]}\left\|\left.\right|_{p, \mu} ^{p}\right\| \varphi(x-k)\right|\right\|_{p, \mu, *}^{p} \mid\|\varphi(x-k)\| \|_{p, \mu}^{p}\right)^{1 / p} \\
\leq & (2 N-1)^{(p-1) / p} C_{3}(2 N-1)^{1 / p}\||f|\|_{p, \mu}
\end{aligned}
$$

et donc $C_{1} \leq(2 N-1) C_{3}$.

Le théorème 2 est donc démontré. 
3. Démonstration de la proposition 1. Commençons par noter pour $R>0, p \in[1, \infty]$ et $\mu$ mesure borélienne positive

$$
\alpha(R)=\sup _{|I|=R} \frac{1}{|I|}\left|\left\|\chi _ { I } \left|\left\|_ { p , \mu } \left|\left\|\chi_{I} \mid\right\|_{p, \mu, *} .\right.\right.\right.\right.\right.
$$

On a le résultat simple suivant:

LEMME 7. Si $R \leq R^{\prime} \leq 2 R$ alors $\frac{1}{2} \alpha(R) \leq \alpha\left(R^{\prime}\right) \leq 16 \alpha(R)^{3}$.

En effet, soit $I=\left[x_{0}-R / 2, x_{0}+R / 2\right]$ un intervalle de longueur $R$ et $I^{\prime}=\left[x_{0}-R^{\prime} / 2, x_{0}+R^{\prime} / 2\right]$. Alors il est clair que $\left\|\chi_{I}\right\|_{p, \mu} \leq\left\|\chi_{I^{\prime}}\right\|_{p, \mu}$ et que $\left\|\left|\chi_{I}\right|\right\|_{p, \mu, *} \leq\left\|\left|\chi_{I^{\prime}}\right|\right\|_{p, \mu, *}$, et donc

$$
\frac{1}{R}\left|\left\|\chi _ { I } \left|\left\|_ { p , \mu } \left|\left\|\chi_{I}||_{p, \mu, *} \leq \frac{R^{\prime}}{R} \frac{1}{R^{\prime}}\left|\left\|\chi _ { I ^ { \prime } } \left|\left\|_ { p , \mu } \left|\left\|\chi_{I^{\prime}} \mid\right\|_{p, \mu, *} \leq 2 \alpha\left(R^{\prime}\right),\right.\right.\right.\right.\right.\right.\right.\right.\right.\right.\right.
$$

d'où $\alpha(R) \leq 2 \alpha\left(R^{\prime}\right)$.

Décomposons maintenant $I^{\prime}$ en $I_{1} \cup I_{2}$ avec

$$
I_{1}=\left[x_{0}-R^{\prime} / 2, x_{0}+R-R^{\prime} / 2\right] \quad \text { et } \quad I_{2}=\left[x_{0}+R^{\prime} / 2-R, x_{0}+R^{\prime} / 2\right] \text {. }
$$

On a

et

$$
||\left|\chi_{I}\right|||_{p, \mu} \leq\left.||\left|\chi_{I_{1}}\right|\right|_{p, \mu}+\left.||\left|\chi_{I_{2}}\right|\right|_{p, \mu}
$$

$$
\left.||\left|\chi_{I}\right|\right|_{p, \mu, *} \leq\left.||\left|\chi_{I_{1}}\right|\right|_{p, \mu, *}+\left.||\left|\chi_{I_{2}}\right|\right|_{p, \mu, *} .
$$

Or

$$
\begin{aligned}
\frac{R}{2} \leq \frac{3 R-R^{\prime}}{2} & =\left|I_{1} \cap I\right| \\
& \leq\|\| \chi_{I_{1}}\left\|\left.\right|_{p, \mu}\right\|\left|\chi_{I}\right|\left\|_{p, \mu, *} \leq \frac{R \alpha(R)}{\left\|\chi_{I_{1}}|\||_{p, \mu, *}\right.}\right\| \chi_{I} \|_{p, \mu, *},
\end{aligned}
$$

d'où

$$
\left\|\left.\left|\chi_{I_{1}}\right|\right|_{p, \mu, *} \leq 2 \alpha(R)||\left|\chi_{I}\right|\right\|_{p, \mu, *} .
$$

On obtient de même

$$
\left\|\chi _ { I _ { 1 } } \left|\left\|_{p, \mu} \leq 2 \alpha(R)\left|\left\|\chi _ { I } | \| _ { p , \mu } , \quad \| | \chi _ { I _ { 2 } } \left|\left\|_{p, \mu} \leq 2 \alpha(R)\left|\left\|\chi_{I} \mid\right\|_{p, \mu}\right.\right.\right.\right.\right.\right.\right.\right.
$$

et

$$
\left\|\left|\chi_{I_{2}}\right|\right\|_{p, \mu, *} \leq 2 \alpha(R)\left|\left\|\chi_{I} \mid\right\|_{p, \mu, *},\right.
$$

et en fin de compte

$$
\begin{aligned}
\frac{1}{R^{\prime}}\left\|\left|\chi_{I^{\prime}}\right|\right\|_{p, \mu}\left|\left\|\chi_{I^{\prime}} \mid\right\|_{p, \mu, *}\right. & \leq \frac{R}{R^{\prime}} 16 \alpha(R)^{2} \frac{1}{R}\left|\left\|\chi _ { I } \left|\left\|_ { p , \mu } \left|\left\|\chi_{I} \mid\right\|_{p, \mu, *}\right.\right.\right.\right.\right. \\
& \leq 16 \alpha(R)^{3} .
\end{aligned}
$$

Le lemme 7 est donc démontré.

LEMME 8. $\left\|\left|P_{j} f\right|\right\|_{p, \mu} \leq(2 N-1)^{2}\|\varphi\|_{\infty}^{2} \alpha\left((2 N-1) / 2^{j}\right)\left|\|f \mid\|_{p, \mu}\right.$. 
En effet, la démonstration du théorème 2 donne

$$
\left.\left\|\left|P_{j} f\right|\right\|\right|_{p, \mu} \leq(2 N-1)\left(\operatorname { s u p } _ { k \in \mathbb { Z } } \left|\left\|\varphi _ { j , k } \left|\left\|\left.\right|_{p, \mu}\left|\left\|\varphi_{j, k} \mid\right\|_{p, \mu, *}\right)|||f|\right\|_{p, \mu} .\right.\right.\right.\right.
$$

Or on a

$$
\left|\varphi_{j, k}(x)\right| \leq 2^{j / 2}\|\varphi\|_{\infty} \chi_{\left[k / 2^{j},(k+2 N-1) / 2^{j}\right]}(x)
$$

et donc

$$
\left.\left.\left\|\left|\varphi_{j, k}\right|\right\|\right|_{p, \mu}\left\|\varphi_{j, k}\right\|\right|_{p, \mu, *} \leq(2 N-1) \alpha\left(\frac{2 N-1}{2^{j}}\right)\|\varphi\|_{\infty}^{2} .
$$

De ce lemme 8 on tire les conclusions suivantes. Si les $\alpha(R)$ sont finis (critère de Muckenhoupt à une échelle), les projecteurs $P_{j}$ sont continus sur $L^{p}(d \mu)$ (et réciproquement d'après le théorème 2 ). Si les $\alpha(R)$ restent bornés quand $R$ tend vers 0 (critère de Muckenhoupt aux petites échelles), les projecteurs $P_{j}$ sont équicontinus pour $j \geq 0$ (et réciproquement, en appliquant le théorème 2 aux mesures $\mu_{j}(x)$ définies par $\left.\int f d \mu_{j}=\int f\left(2^{j} x\right) d \mu\right)$. De même, les $P_{j}, j \in \mathbb{Z}$, sont équicontinus sur $L^{p}(d \mu)$ si et seulement si $\sup _{R} \alpha(R)<\infty$ (critère de Muckenhoupt global).

De plus, si les $P_{j}$ sont équicontinus sur $L^{p}(d \mu)$ pour $j \geq 0$, comme $P_{j} f$ converge vers $f$ dans $L^{p}(d \mu)$ pour $f$ continue à support compact, on a $\lim _{j \rightarrow \infty}\left\|P_{j} f-f\right\|_{p, \mu}=0$ pour tout $f \in L^{p}(d \mu)$. La réciproque est également vraie d'après le théorème de Banach-Steinhaus. De plus, si $E$ est un borélien borné tel que $|E|=0$, alors $P_{j}\left(\chi_{E}\right)=0$ pour tout $j$ et donc, si $\sup _{R \leq 1} \alpha(R)<\infty$, on a $\left\|\chi_{E}\right\|_{p, \mu}=0$ et donc $\mu(E)=0$, ce qui montre que $\mu$ est absolument continue par rapport à la mesure de Lebesgue.

La proposition 1 est donc démontrée. L'équivalence $(\mathrm{B} 1) \Leftrightarrow(\mathrm{B} 2)$ du théorème 1 a été également démontrée. L'équivalence $(\mathrm{A} 1) \Leftrightarrow(\mathrm{A} 2)$ est immédiate : si (A1) est vérifiée, alors les $P_{j}$ sont équicontinus (par Banach-Steinhaus) et donc $v \in A_{p}$; inversement, si $v \in A_{p}$, les $P_{j}$ sont équicontinus et pour tout $f \in L^{p}(d \mu), \lim _{j \rightarrow \infty}\left\|\mid P_{j} f-f\right\|_{p, \mu}=0$. Il reste à vérifier que pour $f \in D, D$ dense dans $L^{p}(d \mu), \lim _{j \rightarrow-\infty}\left\|\mid P_{j} f\right\|_{p, \mu}=0$. Considérons le cas de $f$ bornée à support dans $[-M, M]$; alors on a

$$
\begin{aligned}
\left|P_{j} f(x)\right| \leq & \sum_{k \in \mathbb{Z}} 2^{j}\left|\left\langle f \mid \varphi\left(2^{j} x-k\right)\right\rangle\right|\left|\varphi\left(2^{j} x-k\right)\right| \\
\leq & 2 M\|f\|_{\infty}\|\varphi\|_{\infty}^{2} \\
& \times 2^{j} \sum_{\left[k / 2^{j},(k+2 N-1) / 2^{j}\right] \cap[-M, M] \neq \emptyset} \chi_{\left[k / 2^{j},(k+2 N-1) / 2^{j}\right]}(x) \\
\leq & 2 M\|f\|_{\infty}\|\varphi\|_{\infty}^{2} 2^{j}(2 N-1) \chi_{\left[-M-(2 N-1) / 2^{j}, M+(2 N-1) / 2^{j}\right]}
\end{aligned}
$$

et donc

$$
\left\|\left|P_{j} f\right|\right\|_{p, \mu} \leq C 2^{j}\left|\left\|\chi_{\left[-M-(2 N-1) / 2^{j}, M+(2 N-1) / 2^{j}\right]} \mid\right\|_{p, \mu}\right.
$$




$$
\leq C^{\prime} \frac{1}{\left\|\chi_{\left[-M-(2 N-1) / 2^{j}, M+(2 N-1) / 2^{j}\right]}\right\|_{p, \mu, *}} .
$$

Or on a $d \mu=v d x$ et

$$
\begin{aligned}
& \|\left.\left|\chi_{\left[-M-(2 N-1) / 2^{j}, M+(2 N-1) / 2^{j}\right]}\right|\right|_{p, \mu, *} \\
& =\left(\int_{\left[-M-(2 N-1) / 2^{j}, M+(2 N-1) / 2^{j}\right]} v^{-1 /(p-1)} d x\right)^{(p-1) / p}
\end{aligned}
$$

et il suffit donc de vérifier que si $v \in A_{p}$,

$$
\int_{-\infty}^{\infty} v^{-1 /(p-1)} d x=\infty
$$

Or

$$
\begin{aligned}
&\left(\int_{0}^{2^{N+1}} v^{-1 /(p-1)} d x\right)^{(p-1) / p} \\
&=\left(\int_{0}^{2^{N+1}} v^{-1 /(p-1)} d x\right)^{(p-1) / p} \frac{1}{2^{N}} \int \chi_{\left[0,2^{N+1}\right] \chi_{\left[2^{N}, 2^{N+1}\right]} d x} \\
& \leq \frac{1}{2^{N}}\left(\int_{0}^{2^{N+1}} v^{-1 /(p-1)} d x\right)^{(p-1) / p} \\
& \times\left(\int_{0}^{2^{N+1}} v d x\right)^{1 / p}\left(\int_{2^{N}}^{2^{N+1}} v^{-1 /(p-1)} d x\right)^{(p-1) / p} \\
& \leq C\left(\int_{2^{N}}^{2^{N+1}} v^{-1 /(p-1)} d x\right)^{(p-1) / p}
\end{aligned}
$$

et on voit que

$$
\lim _{N \rightarrow \infty} \int_{0}^{2^{N+1}} v^{-1 /(p-1)} d x=\infty .
$$

On a bien $(\mathrm{A} 1) \Leftrightarrow(\mathrm{A} 2)$. Lorsque $p=1$, (A1) n'est pas vérifié pour $v \in A_{1}$ en général : par exemple pour $v=1$ on a $\int P_{j} f d x=\int f d x$ pour tout $f \in L^{1}(d x)$ (car

$$
\begin{aligned}
\int P_{j} f d x & =\int \sum\left\langle f \mid \varphi_{j, k}\right\rangle \varphi_{j, k} d x=\sum\left\langle f \mid \varphi_{j, k}\right\rangle 2^{-j / 2} \\
& =\int f(x) \sum \varphi\left(2^{j} x-k\right) d x=\int f d x
\end{aligned}
$$

par (7.1)) et donc si $\int f d x \neq 0$ on ne peut avoir $\lim _{j \rightarrow-\infty}\left\|\mid P_{j} f\right\|_{1,1}=0$. 
4. Bases inconditionnelles et opérateurs de Calderón-Zygmund. Nous allons montrer que les ondelettes $\left(\psi_{j, k}\right)$ forment une base inconditionnelle de $L^{p}(v d x)$ lorsque $v \in A_{p}$ et $1<p<\infty$ (nous excluons le cas $p=1$ car les espaces $L^{1}(v d x)$ n'admettent pas de bases inconditionnelles). De même, la famille $\left(\varphi_{j_{0}, k}\right)_{k \in \mathbb{Z}} \cup\left(\psi_{j, k}\right)_{j \geq j_{0}, k \in \mathbb{Z}}$ forme une base inconditionnelle de $L^{p}(v d x)$ si et seulement si $v$ satisfait le critère local (ou aux petites échelles) de Muckenhoupt.

Proposition 2. Soit $\left(V_{j}\right)$ une analyse multi-résolution d'I. Daubechies (à ondelettes höldériennes à support compact), $v$ une fonction de poids sur $\mathbb{R}(v \geq 0$ p.p., $v$ localement intégrable $)$ et $p \in] 1, \infty[$. Alors :

(i) Les assertions suivantes sont équivalentes:

(D1) les $\psi_{j, k}, j \in \mathbb{Z}, k \in \mathbb{Z}$, forment une base inconditionnelle de $L^{p}(v d x)$;

(D2) la norme $\|f \mid\|_{p, v}$ est équivalente à la norme

$$
N_{p, v}(f)=\|||\left(\sum_{j \in \mathbb{Z}} \sum_{k \in \mathbb{Z}}\left|\left\langle f \mid \psi_{j, k}\right\rangle\right|^{2} \chi_{j, k}(x)^{2}\right)^{1 / 2}||_{p, v}
$$

(D3) $v \in A_{p}$

$$
\text { ò̀ } \chi_{0}=\chi_{[0,1]} \text { et } \chi_{j, k}(x)=2^{j / 2} \chi\left(2^{j} x-k\right) \text {; }
$$

(ii) Les assertions suivantes sont équivalentes:

(E1) les $\left(\varphi_{j_{0}, k}\right), k \in \mathbb{Z}$, et les $\left(\psi_{j, k}\right), j \geq j_{0}, k \in \mathbb{Z}$, forment une base inconditionnelle de $L^{p}(v d x)$;

(E2) la norme $\|f f\|_{p, v}$ est équivalente à la norme

$$
\begin{aligned}
M_{p, v, j_{0}}(f)= & \left(\sum_{k \in \mathbb{Z}}\left|\left\langle f \mid \varphi_{j_{0}, k}\right\rangle\right|^{p}\left|\left\|\varphi_{j_{0}, k} \mid\right\|_{p, v}^{p}\right)^{1 / p}\right. \\
& +\left\|\mid\left(\sum_{j \geq j_{0}} \sum_{k}\left|\left\langle f \mid \psi_{j, k}\right\rangle\right|^{2} \chi_{j, k}(x)^{2}\right)^{1 / 2}\right\| \|_{p, v} ;
\end{aligned}
$$

(E3) $\sup _{|I| \leq 1}|I|^{-1}||\left|\chi_{I}\right|\left\|_{p, v}|| \chi_{I} \mid\right\|_{p, v, *}<\infty$.

Rappelons qu'une suite $\left(b_{n}\right)_{n \in \mathbb{N}}$ est une base inconditionnelle d'un espace de Banach $B$ si :

(i) tout élément $b$ de $B$ s'écrit $b=\lim _{N \rightarrow \infty} \sum_{k=0}^{N} \beta_{k}(b) b_{k}$, la convergence ayant lieu dans $B$ et les coefficients $\beta_{k}(b)$ étant uniques;

(ii) il existe une constante $C$ telle que

$$
\forall b \in B, \forall \varepsilon \in\{-1,1\}^{\mathbb{N}}, \quad\left\|\sum_{n \in \mathbb{N}} \varepsilon(n) \beta_{n}(b) b_{n}\right\|_{B} \leq C\|b\|_{B} .
$$


Les familles $\left(\varepsilon(n) \beta_{n}(b) b_{n}\right)_{n \in \mathbb{N}}$ sont alors sommables dans $B$ et les séries sont commutativement convergentes, de sorte que la façon d'indexer la famille $\left(b_{n}\right)$ est indifférente.

Si $\left(b_{n}\right)_{n \in \mathbb{N}}$ est une base inconditionnelle de $B$ et si $A \subset \mathbb{N}$, l'opérateur de somme partielle $b \rightarrow P_{A}(b)=\sum_{n \in A} \beta_{n}(b) b_{n}$ est continu et sa norme d'opérateur se majore indépendamment de $A$, puisque $2 P_{A}(b)-b=$ $\sum_{n \in \mathbb{N}} \varepsilon_{A}(n) \beta_{n}(b) b_{n}$ avec $\varepsilon_{A}(n)=1$ si $n \in A$ et $\varepsilon_{A}(n)=-1$ si $n \notin A$.

On en conclut immédiatement que (D2) $\Rightarrow(\mathrm{D} 1)$, que (D1) implique que les opérateurs $P_{j}$ sont équicontinus (car on a

$$
P_{j} f=\sum_{l<j} \sum_{k}\left\langle f \mid \psi_{l, k}\right\rangle \psi_{l, k}
$$

et donc $P_{j}$ est un opérateur de somme partielle) et donc que (D1) $\Rightarrow(\mathrm{D} 3)$. De même $(\mathrm{E} 2) \Rightarrow(\mathrm{E} 1)$ et $(\mathrm{E} 1) \Rightarrow(\mathrm{E} 3)$.

On est donc ramené à montrer $(\mathrm{D} 3) \Rightarrow(\mathrm{D} 2)$ et $(\mathrm{E} 3) \Rightarrow(\mathrm{E} 2)$. En fait, il suffit de montrer que $v \in A_{p}$ implique

$$
\left\|\left|\left(\sum_{j \in \mathbb{Z}} \sum_{k \in \mathbb{Z}}\left|\left\langle f \mid \psi_{j, k}\right\rangle\right|^{2} \chi_{j, k}(x)^{2}\right)^{1 / 2}\|\|_{p, v} \leq C_{p, v}\|\mid f\|_{p, v}\right.\right.
$$

et, de même, que $\sup _{|I| \leq 1}|I|^{-1}||\left|\chi_{I}\right|\left\|_{p, v}\left|\left\|\chi_{I} \mid\right\|_{p, v, *}<\infty\right.\right.$ implique que

$$
\begin{gathered}
\left(\sum_{k \in \mathbb{Z}}\left|\left\langle f \mid \varphi_{j_{0}, k}\right\rangle\right|^{p} \|\left.\left|\varphi_{j_{0}, k}\right|\right|_{p, v} ^{p}\right)^{1 / p} \leq C_{j_{0}, p, v}\||f|\|_{p, v}, \\
\left\|\left|\left(\sum_{j \geq j_{0}} \sum_{k \in \mathbb{Z}}\left|\left\langle f \mid \psi_{j, k}\right\rangle\right|^{2} \chi_{j, k}(x)^{2}\right)^{1 / 2}\left\|\left.\right|_{p, v} \leq C_{j_{0}, p, v}\right\|\right| f \mid\right\|_{p, v} .
\end{gathered}
$$

Les inégalités réciproques s'obtiennent par dualité, car $\langle\mid\rangle$ identifie le dual de $L^{p}(v d x)$ à $L^{p *}\left(v^{*} d x\right)$ avec $p^{*}=p /(p-1)$ et $v^{*}=v^{-1 /(p-1)}$; de plus,

$$
\left\|\left.\left|\chi_{I}\right|\right|_{p, v}=\left.\left|\| \chi_{I}\right|\right|_{p^{*}, v^{*}, *} \text { et } \quad\right\| \chi_{I}\left\|_{p, v, *}=\right\|\left|\chi_{I}\right| \|_{p, v}
$$

de sorte que (9) (ou (10)) est également vérifiée pour $p^{*}, v^{*}$, et donc

$$
\begin{aligned}
& \|\| f \mid \|_{p, v}=\sup \left\{|\langle f \mid g\rangle|: g \in L^{p *}\left(v^{*} d x\right),\left\|\left|\|g \mid\|_{p^{*}, v^{*}} \leq 1\right\}\right.\right. \\
& =\sup \left\{\left|\sum_{j, k}\right|\left\langle f \mid \psi_{j, k}\right\rangle\left\langle\psi_{j, k} \mid g\right\rangle \mid: g \in L^{p *}\left(v^{*} d x\right),\|g\|_{p^{*}, v^{*}} \leq 1\right\} \\
& \leq \sup \left\{\sum_{j, k}\left|\left\langle f \mid \psi_{j, k}\right\rangle\right|\left|\left\langle\psi_{j, k} \mid g\right\rangle\right| \int \chi_{j, k}^{2} d x: g \in L^{p *}\left(v^{*} d x\right),\|\| g \|_{p^{*}, v^{*}} \leq 1\right\} \\
& \leq \sup \left\{\int\left(\sum_{j, k}\left|\left\langle f \mid \psi_{j, k}\right\rangle\right|^{2} \chi_{j, k}(x)^{2}\right)^{1 / 2}\right. \\
& \left.\quad \times\left(\sum_{j, k}\left|\left\langle g \mid \psi_{j, k}\right\rangle\right|^{2} \chi_{j, k}(x)^{2}\right)^{1 / 2} d x:\||| \mid\|_{p^{*}, v^{*}} \leq 1\right\}
\end{aligned}
$$




$$
\leq N_{p, v}(f) \sup \left\{N_{p^{*}, v^{*}}(g):\|g\|_{p^{*}, v^{*}} \leq 1\right\} \leq C_{p^{*}, v^{*}} N_{p, v}(f)
$$

(et de même on contrôle $\|f f\|_{p, v}$ par $M_{p, v}(f)$ grâce à (10) appliquée à $p^{*}, v^{*}$ ).

On est donc ramené à établir (9) et (10). (10.1) est immédiat puisque

$$
\left|\left\langle f \mid \varphi_{j_{0}, k}\right\rangle\right|^{p} \leq||\left|\varphi_{j_{0}, k}\right| \|_{p, v, *}^{p} \int_{\left[k / 2^{j_{0}},(k+2 N-1) / 2^{j_{0}}\right]}|f|^{p} v d x
$$

et qu'on a vu que $\sup _{k}\left|\left\|\varphi_{j_{0}, k}\left|\left\|_{p, v}||\left|\varphi_{j_{0}, k}\right|\right\|_{p, v, *}\right.\right.\right.$ était borné en fonction de

$$
\sup _{|I| \leq 1} \frac{1}{|I|}\left\|\chi _ { I } \left|\left\|_ { p , v } \left|\left\|\chi_{I} \mid\right\|_{p, v, *} .\right.\right.\right.\right.
$$

Par ailleurs on note $\omega$ une fonction $\mathcal{C}^{\infty}$ à support dans $[-1,2]$ et valant sur $[0,1]$. On va montrer que

$$
\sup _{\varepsilon}\left|\left\|\sum_{j} \sum_{k} \varepsilon(j, k)\left\langle f \mid \psi_{j, k}\right\rangle \omega_{j, k}\left|\left\|_{p, v} \leq C_{p, v}\right\| f\right|\right\|_{p, v}\right.
$$

où $\varepsilon \in\{-1,1\}^{\mathbb{Z}^{2}}, \omega_{j, k}(x)=2^{j / 2} \omega\left(2^{j} x-k\right)$ et $v \in A_{p}$, et de même,

$$
\sup _{\varepsilon}\left\|\left|\sum_{j \geq j_{0}} \sum_{k} \varepsilon(j, k)\left\langle f \mid \psi_{j, k}\right\rangle \omega_{j, k}\right|\right\|_{p, v} \leq C_{p, v}\|f f\|_{p, v}
$$

lorsque $\sup _{|I| \leq 1}|I|^{-1}\left|\left\|\chi_{I}\left|\left\|_{p, v}\right\| \chi_{I}\right|\right\|_{p, v, *}<\infty\right.$. Si on moyenne (9bis) sur tous les choix possibles de $\varepsilon$, on obtient, par l'inégalité de Khintchine,

$$
\begin{aligned}
\int_{\{-1,1\}^{\mathbb{Z}^{2}}}\left|\sum_{j} \sum_{k} \varepsilon(j, k)\left\langle f \mid \psi_{j, k}\right\rangle \omega_{j, k}(x)\right|^{p} d \varepsilon & \\
& \geq \gamma_{p}\left(\sum_{j} \sum_{k}\left|\left\langle f \mid \psi_{j, k}\right\rangle\right|^{2} \omega_{j, k}(x)^{2}\right)^{p / 2}
\end{aligned}
$$

et donc

$$
\gamma_{p} \int\left(\sum_{j} \sum_{k}\left|\left\langle f \mid \psi_{j, k}\right\rangle\right|^{2} \omega_{j, k}(x)^{2}\right)^{p / 2} v d x \leq C_{p, v}^{p}\|\| f \|_{p, v}^{p} ;
$$

comme $\left|\omega_{j, k}(x)\right| \geq \chi_{j, k}(x)$, (9) se déduit de (9bis) et de même (10) se déduit de (10bis).

On conclut alors grâce à la théorie des opérateurs de Calderón-Zygmund [7]. Si $T$ est un opérateur linéaire continu de $L^{2}(\mathbb{R}, d x)$ dans $L^{2}(\mathbb{R}, d x)$ tel que le noyau-distribution $K(x, y)$ de $T$ coïncide en dehors de la diagonale $x=y$ avec une fonction continue localement höldérienne (d'exposant $\alpha>0$ ) telle que

$$
|K(x, y)| \leq C_{1} \frac{1}{|x-y|},
$$




$$
|K(x, y)-K(x+z, y)|+|K(x, y)-K(x, y+z)| \leq C_{2} \frac{|z|^{\alpha}}{|x-y|^{1+\alpha}}
$$

pour $z<\frac{1}{2}|x-y|$,

$$
\|T f\|_{2} \leq C_{3}\|f\|_{2}
$$

et si $v \in A_{p}$,

$$
\forall I \quad \frac{1}{|I|}\left|\left\|\chi _ { I } | | _ { p , v } \left|\left\|\chi_{I} \mid\right\|_{p, v, *} \leq C_{4}\right.\right.\right.
$$

pour un $p \in] 1, \infty[$, alors on a

$$
\|T f\|_{p, v} \leq C_{5} \mid\|f\|_{p, v}
$$

où $C_{5}$ ne dépend que de $\alpha, C_{1}, C_{2}, C_{3}, C_{4}$ et $p$.

(9bis) est alors immédiat. On note $T_{\varepsilon}$ l'opérateur

$$
f \rightarrow \sum \varepsilon_{(j, k)}\left\langle f \mid \psi_{j, k}\right\rangle \omega_{j, k}
$$

et $K_{\varepsilon}$ son noyau. En dehors de la diagonale, on a

$$
K_{\varepsilon}(x, y)=\sum \varepsilon_{(j, k)} \psi_{j, k}(y) \omega_{j, k}(x)
$$

et donc

$$
\left|K_{\varepsilon}(x, y)\right| \leq \sum_{j} \sum_{k} 2^{j}\left|\psi\left(2^{j} y-k\right)\right| \cdot\left|\omega\left(2^{j} x-k\right)\right| .
$$

Or $\psi\left(2^{j} y-k\right)$ est non nul seulement si $2^{j} y-2 N+1<k<2^{j} y$ et de même $\omega\left(2^{j} x-k\right)$ est non nul seulement si $2^{j} x-2<k<2^{j} x+1$; le produit est non nul seulement si $-2<2^{j}(y-x)<2 N$ et donc $2^{j}|y-x| \leq 2 N$. On a alors

$$
\left|K_{\varepsilon}(x, y)\right| \leq \sum_{2^{j}|y-x| \leq 2 N} 2^{j}\|\psi\|_{\infty}\|\omega\|_{\infty} \cdot 3 \leq \frac{C}{|x-y|} .
$$

De même on a

$$
\begin{aligned}
\mid K_{\varepsilon}(x, y) & -K_{\varepsilon}(x, z+y) \mid \\
& \leq \sum_{j} \sum_{k} 2^{j}\left|\omega\left(2^{j} x-k\right)\right| \cdot\left|\psi\left(2^{j} y-k\right)-\psi\left(2^{j}(z+y)-k\right)\right| .
\end{aligned}
$$

Or $\psi$ est höldérienne d'exposant $\beta>0$, de sorte qu'on a

$$
\left|\psi\left(2^{j} y-k\right)-\psi\left(2^{j}(y+z)-k\right)\right| \leq C 2^{j \beta}|z|^{\beta} .
$$

La somme court à nouveau pour $2^{j}|y-x| \leq 4 N$ si $|z| \leq \frac{1}{2}|x-y|$ et on obtient

$$
\begin{aligned}
\left|K_{\varepsilon}(x, y)-K_{\varepsilon}(x, y+z)\right| & \leq \sum_{2^{j}|y-x| \leq 4 N} 2^{j} C 2^{j \beta}|z|^{\beta}\|\omega\|_{\infty} \cdot 3 \\
& \leq C^{\prime} \frac{|z|^{\beta}}{|x-y|^{1+\beta}} \quad \text { pour }|z|<\frac{1}{2}|x-y| .
\end{aligned}
$$


On obtient de même

$$
\left|K_{\varepsilon}(x, y)-K_{\varepsilon}(x+z, y)\right| \leq C^{\prime} \frac{|z|^{\beta}}{|x-y|^{1+\beta}} \quad \text { pour }|z|<\frac{1}{2}|x-y| .
$$

Enfin, il suffit de vérifier que

$$
\left\|\sum_{j, k} \lambda_{j, k} \omega_{j, k}\right\|_{2}^{2} \leq C \sum_{j, k}\left|\lambda_{j, k}\right|^{2}
$$

pour obtenir

$$
\left\|T_{\varepsilon} f\right\|_{2}^{2} \leq C \sum_{j, k}\left|\left\langle f \mid \psi_{j, k}\right\rangle\right|^{2} \leq C\|f\|_{2}^{2} .
$$

Or cette inégalité est élémentaire (voir [2] par exemple).

Les $T_{\varepsilon}, \varepsilon \in\{-1,1\}^{\mathbb{Z}^{2}}$, vérifient donc (11.1) à (11.4) uniformément; ils sont donc équicontinus sur $L^{p}(v d x)$ lorsque $v \in A_{p}$ et (9bis) est donc démontré.

Pour démontrer (10bis), posons $S_{\varepsilon}=T_{\varepsilon} \circ\left(\mathrm{Id}-P_{j_{0}}\right)$ :

$$
S_{\varepsilon} f=\sum_{j \geq j_{0}} \sum_{k} \varepsilon(j, k)\left\langle f \mid \psi_{j, k}\right\rangle \omega_{j, k} .
$$

Il est clair qu'à nouveau les $S_{\varepsilon}, \varepsilon \in\{-1,1\}^{\mathbb{Z}^{2}}$, vérifient (11.1) à (11.4) uniformément. De plus le noyau $G_{\varepsilon}(x, y)$ de $S_{\varepsilon}$ vérifie

$$
G_{\varepsilon}(x, y)=0 \quad \text { si }|x-y| \geq 2 N \cdot(1 / 2)^{j_{0}} .
$$

Supposons maintenant que $v$ satisfasse le critère de Muckenhoupt aux petites échelles :

$$
\sup _{|I| \leq 1} \frac{1}{|I|}\left|\left\|\chi _ { I } \left|\left\|_{p, v}||\left|\chi_{I}\right|\right\|_{p, v, *}<\infty\right.\right.\right.
$$

On considère $f \in L^{p}(v d x)$ et on pose $f_{k}=f \chi_{\left[k / 2^{j_{0}},(k+1) / 2^{j_{0}}\right]}$. On va montrer que

$$
\left\|\left.\left|S_{\varepsilon} f_{k}\right|\right|_{p, v} \leq C \mid\right\| f_{k} \|_{p, v}
$$

où $C$ est indépendante de $\varepsilon$ et de $k$. Comme Supp $S_{\varepsilon} f_{k} \subset\left[(k-2 N) / 2^{j_{0}}\right.$, $\left.(k+2 N+1) / 2^{j_{0}}\right]$, on obtient alors :

$$
\begin{aligned}
\left\|S_{\varepsilon} f\right\|_{p, v} & \leq \sum_{r=0}^{4 N}\|\| \sum_{k \in \mathbb{Z}} S_{\varepsilon} f_{r+(4 N+1) k}\|\|_{p, v} \\
& \leq(4 N+1)^{(p-1) / p}\left(\left.\sum_{k \in \mathbb{Z}}\left\|S_{\varepsilon} f_{k}\right\|\right|_{p, v} ^{p}\right)^{1 / p} \\
& \leq C(4 N+1)^{(p-1) / p}\|\mid f\|_{p, v}
\end{aligned}
$$

et (10bis) sera démontré.

Pour estimer $\left\|\mid S_{\varepsilon} f_{k}\right\|_{p, v}$ on définit le poids $v_{k}$ par : 
(i) $v_{k}=v \operatorname{sur}\left[\frac{k-2 N}{2^{j_{0}}}, \frac{k+2 N+1}{2^{j_{0}}}\right]$,

(ii) $v_{k}(x)=v\left(2 \frac{k+2 N+1}{2^{j_{0}}}-x\right) \operatorname{sur}\left[\frac{k+2 N+1}{2^{j_{0}}}, \frac{k+6 N+2}{2^{j_{0}}}\right]$,

(iii) $v_{k}$ est étendu en dehors de $\left[(k-2 N) / 2^{j_{0}},(k+6 N+2) / 2^{j_{0}}\right]$ par périodicité de période $(8 N+2) / 2^{j_{0}}$.

Alors :

(j) $\mid\left\|S_{\varepsilon} f_{k}\right\|_{p, v_{k}}=\|\| S_{\varepsilon} f_{k} \|_{p, v}$ et $\left\|f_{k}\right\|_{p, v_{k}}=\|\| f_{k}\|\|_{p, v}$,

(jj) $\sup _{k} \sup _{I}|I|^{-1}\left|\left\|\chi_{I}\left|\left\|_{p, v_{k}}\right\| \chi_{I}\right|\right\|_{p, v_{k}, *}<\infty\right.$.

En effet, si $|I| \geq(8 N+2) / 2^{j_{0}}$, alors si

$$
2^{q} \frac{8 N+2}{2^{j_{0}}} \leq|I| \leq 2^{q+1} \frac{8 N+2}{2^{j_{0}}},
$$

on a

$$
\begin{aligned}
& 2^{q / p}\left|\left\|\chi_{\left[0,(8 N+2) / 2^{j_{0}}\right]}\left|\left\|_{p, v_{k}} \leq\right\|\left\|\chi_{I}\right\|\left\|_{p, v_{k}} \leq 2^{(q+1) / p}\right\| \chi_{\left[0,(8 N+2) / 2^{j_{0}}\right]}\right|\right\|_{p, v_{k}},\right. \\
& 2^{q(p-1) / p} \| \chi_{\left[0,(8 N+2) / 2^{j_{0}}\right]}||_{p, v_{k}, *} \\
& \leq||\left|\chi_{I}\right|\left\|_{p, v_{k}, *} \leq 2^{(q+1)(p-1) / p}||\left|\chi_{\left[0,(8 N+2) / 2^{j_{0}}\right]}\right|\right\|_{p, v_{k}, *}
\end{aligned}
$$

et

$\left\||| \chi_{\left[0,(8 N+2) / 2^{j_{0}}\right]} \mid\right\|_{p, v_{k}}=2^{1 / p}\left\|\chi_{\left[(k-2 N) / 2^{j_{0}},(k+2 N+1) / 2^{j_{0}}\right]}\right\|_{p, v}$,

$\left\|\left|\chi_{\left[0,(8 N+2) / 2^{j_{0}}\right]}\right|\right\|_{p, v_{k}, *}=2^{(p-1) / p}\left|\left\|\chi_{\left[(k-2 N) / 2^{j_{0}},(k+2 N+1) / 2^{j_{0}}\right]} \mid\right\|_{p, v, *}\right.$

et enfin,

$$
\begin{aligned}
\frac{1}{|I|}\left\|\chi_{I}\left|\left\|_{p, v_{k}}\right\| \chi_{I}\right|\right\|_{p, v_{k}, *} \leq & \frac{2^{j_{0}+2}}{8 N+2}\left\|\chi_{\left[(k-2 N) / 2^{j_{0}},(k+2 N+1) / 2^{j_{0}}\right]} \mid\right\|_{p, v} \\
& \times\left.\left\|\chi_{\left[(k-2 N) / 2^{j_{0}},(k+2 N+1) / 2^{j_{0}}\right]}\right\|\right|_{p, v, *} \\
\leq & 2 \alpha\left(\frac{4 N+1}{2^{j_{0}}}\right) .
\end{aligned}
$$

Supposons maintenant $|I| \leq(8 N+2) / 2^{j_{0}}$. Trois cas sont alors possibles :

(i) $I$ est entièrement contenu dans un translaté de

$$
\left[\frac{k-2 N}{2^{j_{0}}}, \frac{k+2 N+1}{2^{j_{0}}}\right] \text { ou }\left[\frac{k+2 N+1}{2^{j_{0}}}, \frac{k+6 N+2}{2^{j_{0}}}\right] .
$$

Alors $|I|^{-1}||\left|\chi_{I}\right|\left\|_{p, v_{k}}|| \chi_{I} \mid\right\|_{p, v_{k}, *}$ se contrôle immédiatement par $\alpha(|I|)$.

(ii) $I$ contient un translaté de

$$
\left[\frac{k-2 N}{2^{j_{0}}}, \frac{k+2 N+1}{2^{j_{0}}}\right] \text { ou }\left[\frac{k+2 N+1}{2^{j_{0}}}, \frac{k+6 N+2}{2^{j_{0}}}\right] .
$$


Alors $|I| \geq(4 N+1) / 2^{j_{0}}$ tandis que

$$
\left\||| \chi_{I}||_{p, v_{k}} \leq||\left|\chi_{\left[0,(8 N+2) / 2^{j_{0}}\right]}\right|\right\|_{p, v_{k}}
$$

et

$$
\left.||\left|\chi_{I}\right|\right|_{p, v_{k}, *} \leq\left.||\left|\chi_{\left[0,(8 N+2) / 2^{j} j_{0}\right]}\right|\right|_{p, v_{k}, *} .
$$

On obtient alors

$$
\begin{aligned}
& \frac{1}{|I|}\left|\left\|\chi _ { I } \left|\left\|_{p, v_{k}}||\left|\chi_{I}\right|\right\|_{p, v_{k}, *}\right.\right.\right. \\
& \quad \leq 2 \frac{1}{\left|\left[0,(8 N+2) / 2^{j_{0}}\right]\right|}\left|\left\|\chi_{\left[0,(8 N+2) / 2^{j_{0}}\right]}\left|\left\|_{p, v_{k}}\right\| \chi_{\left[0,(8 N+2) / 2^{j_{0}}\right]}\right|\right\|_{p, v_{k}, *}\right. \\
& \quad \leq 4 \alpha\left(\frac{4 N+1}{2^{j_{0}}}\right) .
\end{aligned}
$$

(iii) Dans le cas contraire, on a $I=\tilde{I} \cup \tilde{\tilde{I}}$ où $\tilde{I}$ et $\tilde{\tilde{I}}$ ont une extrémité commune et sont contenus dans des translatés de

$$
\left[\frac{k-2 N}{2^{j_{0}}}, \frac{k+2 N+1}{2^{j_{0}}}\right] \text { et }\left[\frac{k+2 N+1}{2^{j_{0}}}, \frac{k+6 N+2}{2^{j_{0}}}\right] .
$$

$\operatorname{Si}|\tilde{I}| \geq|\tilde{\tilde{I}}|$, on a

$$
\left\|\left.\left|\chi_{\tilde{I}}\right|\right|_{p, v_{k}} \leq||\left|\chi_{\tilde{I}}\right|\right\|_{p, v_{k}}, \quad||\left|\chi_{\tilde{I}}\right|\left\|_{p, v_{k}, *} \leq||\left|\chi_{\tilde{I}}\right|\right\|_{p, v_{k}, *},
$$

et donc

$$
\frac{1}{|I|}||\left|\chi_{I}\right|\left\|\left._{p, v_{k}}||\left|\chi_{I}\right|\right|_{p, v_{k}, *} \leq \frac{2}{|I|}\left|\left\|\chi _ { \tilde { I } } \left|\left\|_{p, v_{k}}|| \chi_{\tilde{I}} \mid\right\|_{p, v_{k}, *} \leq 4 \alpha(|\tilde{I}|) .\right.\right.\right.\right.
$$

$\mathrm{Au}$ total,

$$
\sup _{I} \frac{1}{|I|}||\left|\chi_{I}\right|||_{p, v_{k}}||\left|\chi_{I}\right|\left\|_{p, v_{k}, *} \leq 4 \sup _{|I| \leq(4 N+1) / 2^{j} 0} \frac{1}{|I|}||\left|\chi_{I}\right|\right\|_{p, v}||\left|\chi_{I}\right| \|_{p, v, *} .
$$

Les $v_{k}$ sont donc uniformément dans la classe $A_{p}$ et on a bien

$$
\left\|S_{\varepsilon} f_{k}\right\|_{p, v_{k}} \leq C \mid\|f\|_{p, v_{k}}
$$

uniformément par rapport à $\varepsilon$ et à $k$.

La proposition 2 est donc démontrée.

\section{Bibliographie}

[1] I. Daubechies, Orthonormal basis of compactly supported wavelets, Comm. Pure Appl. Math. 41 (1988), 909-996.

[2] P. G. Lemarié, Fonctions à support compact dans les analyses multi-résolutions, Rev. Mat. Iberoamericana 7 (1991), 157-182.

[3] P. G. Lemarié et G. Malgouyres, Support des fonctions de base dans une analyse multi-résolution, C. R. Acad. Sci. Paris 313 (1991), 377-380. 
[4] G. Malgouyres, Analyse multi-résolution sur l'intervalle: algorithmes rapides, preprint, Univ. Paris-XI, 1991.

[5] S. Mallat, Multiresolution approximation and wavelet bases of $L^{2}(\mathbb{R})$, Trans. Amer. Math. Soc. 315 (1989), 69-87.

[6] Y. Meyer, Ondelettes et opérateurs, tome I, Hermann, Paris, 1990.

[7] —, Ondelettes et opérateurs, tome II, Hermann, Paris, 1991.

UA CNRS 757

UNIVERSITÉ DE PARIS-SUD

MATHÉMATIQUES

BÂTIMENT 425

91405 ORSAY CEDEX, FRANCE 